\title{
Bedeutung des Neurotoxins MPTP für Ätiologie und Therapie der idiopathischen Parkinsonkrankheit*
}

\author{
K.W. Lange \\ University Department of Neurology \& Parkinson's Disease Society Research Centre, Institute of Psychiatry, London
}

Significance of the neurotoxin MPTP for aetiology and therapy of idiopathic Parkinson's disease

Exposure of drug addicts to MPTP (1-methyl4-phenyl-1,2,3,6-tetrahydropyridine) has caused a Parkinsonian syndrome accompanied by a selective destruction of dopamine containing neurones in the pars compacta of the substantia nigra. MPTP in the human causes a severe irreversible state that very closely resembles idiopathic Parkinson's disease both in its clinical features and response to pharmacological treatment.

Interest in potential environmental agents that might play a role in the aetiology of idiopathic Parkinson's disease is likely to increase as the result of the discovery of the relatively simple molecule MPTP which is highly toxic to the substantia nigra.

Until the discovery of the neurotoxicity of MPTP there was no effective animal model of Parkinson's disease. Administration of PTP to monkeys induces persistent parkinsonism which responds to classical antiparkinsonian therapy. The morphological and biochemical changes in the brains of the animals are more limited and selective than those seen in idiopathic Parkinson's disease. The model of MPTPtreated monkeys appears to provide a useful testbed for the evaluation of future treatments for the disease.

The precise mechanism of MPTP toxicity has yet to be determined and may provide the clue to the mechanism of neuronal death in Parkinson's disease. After entering the brain MPTP is oxidized to MPP $^{+}$(1-methyl-4phenylpyridine) at an extraneuronal site. $\mathrm{MPP}^{+}$is then taken up into dopamine neurones where it accumulates causing cell death by an unknown mechanism. The conversion of MPTP to $\mathrm{MPP}^{+}$is effected by monoamine oxidase B (MAO B). MAO B inhibition using compounds such as L-deprenyl as an adjunct to conventional L-dopa therapy may arrest to some extent the course of Parkinson's disease and may increase life expectancy.

\section{Einleitung}

Die idiopathische Parkinsonkrankheit ist klinisch gekennzeichnet durch Akinesie, Rigor und Tremor. Patho-

\section{Zusammenfassung}

Der Kontakt von Drogenabhängigen mit MPTP (1-Methyl-4-phenyl-1,2,3,6-tetrahydropyridin) verursachte ein Parkinsonsyndrom, das von selektiver Zerstörung dopaminhaltiger Neurone in der Pars compacta der Substantia nigra begleitet wird. MPTP ruft beim Menschen einen dauerhaften Zustand hervor, der hinsichtlich der klinischen Symptome wie auch der Reaktion auf pharmakotherapeutische Maß3nahmen in hohem Maße der idiopathischen Parkinsonkrankheit ähnelt.

Das Interesse an Substanzen in der Umwelt, die möglicherweise eine ätiologische Bedeutung für die idiopathische Parkinsonkrankheit haben, wird als Folge der Entdeckung des vergleichsweise einfachen Moleküls MPTP mit seiner starken Toxizität für die Substantia nigra wahrscheinlich zunehmen.

Vor der Entdeckung der Neurotoxizität von MPTP gab es kein wirkungsvolles Tiermodell der Parkinsonkrankheit. Die Gabe von MPTP bei Affen führt ein anhaltendes Parkinsonsyndrom herbei, das auf klassische Antiparkinsontherapie anspricht. Die morphologischen und biochemischen Veränderungen in den Gehirnen der Tiere sind begrenzter und selektiver als die bei idiopathischer Parkinsonkrankheit. Das Modell der mit MPTP behandelten Affen scheint ein nützliches Instrument für die Beurteilung künftiger Behandlungsverfahren für die Erkrankung zu sein.

Der genaue Mechanismus der Toxizität von MPTP muß noch bestimmt werden und kann vielleicht den Schlüssel für das Verständnis des Neuronenuntergangs bei der Parkinsonkrankheit liefern. Nach der Aufnahme in das Gehirn wird MPTP extraneuronal zu $\mathrm{MPP}^{+}$(1-Methyl-4phenylpyridin) oxidiert. $\mathrm{MPP}^{+}$wird dann in dopaminerge Neurone aufgenommen, wo es angehäuft wird und auf unbekannte Weise den Zelltod verursacht. Die Umwandlung von MPTP zu MPP ${ }^{+}$wird durch Monoaminoxidase B (MAO B) herbeigeführt. Die Hemmung von MAO B beispielsweise durch L-Deprenyl unterdrückt die Toxizität von MPTP. Die Gabe von L-Deprenyl über längere Zeit zusätzlich zur üblichen L-Dopa-Therapie kann möglicherweise das Fortschreiten der Parkinsonkrankheit bis zu einem gewissen Grade hemmen und die Lebenserwartung erhöhen. logische Veränderungen bei dieser Erkrankung zeigen dic meisten pigmentierten Hirnstammkerne. Vor allem die Pars com-

* Mit Unterstützung durch die Deutsche Forschungsgemeinschaft 
pacta der Substantia nigra und der Locus coeruleus weisen einen Untergang melaninhaltiger Neurone auf. Weniger regelmäßig sind viele andere Hirnregionen betroffen (Forno, 1982). Kugelige eosinophile Zytoplasmaeinschlüsse, die Lewykörper genannt werden, sind ein charakteristisches neuropathologisches Merkmal der Parkinsonkrankheit. Die biochemischen Veränderungen in den Gehirnen von Parkinsonkranken betreffen viele Überträgersubstanzen; insbesondere die Dopaminkonzentration ist deutlich vermindert (Hornykiewicz, 1982). Verluste an Überträgerstoffen werden auch für Noradrenalin, Serotonin, Azetylcholin, Gamma-Amino-Buttersäure (Hornykiewicz, 1982; Rinne, 1982) und einige Neuropeptide (Javoy-Agid et al., 1984) beschrieben. Der wichtigste pathochemische Befund bei der Parkinsonkrankheit ist der Verlust dopaminhaltiger Neurone in der Substantia nigra pars compacta mit daraus folgender starker Dopaminverringerung in Nucleus caudatus und Putamen. Die besondere Bedeutung des Dopaminmangels wird dadurch belegt, da $B$ die motorischen Störungen von Parkinsonpatienten nach Dopaminersatz durch die therapeutische Gabe von LDopa, einer Vorstufe des Dopamins, oder von Dopaminagonisten, die die Dopaminrezeptoren unmittelbar stimulieren, aufgehoben werden können.

Trotz umfangreicher Kenntnisse über morphologische und biochemische Veränderungen sowie pharmakotherapeutische Möglichkeiten bei der Parkinsonkrankheit bleiben viele Fragen unbeantwortet. Völlig unklar ist die Ursache für das Sterben eines bestimmten neuronalen Systems und das Auftreten von Lewykörpern. Es ist nicht bekannt, ob die Veränderungen in nicht-dopaminergen Neuronensystemen eine primäre Folge der Krankheit oder eine sekundäre Auswirkung des Todes dopaminhaltiger Neurone sind. In klinischer Hinsicht ist beispielsweise offen, warum die Wirksamkeit von L-Dopa bei langfristiger Behandlung nachläßt. Ein Grund für das mangelhafte Verständnis dieser Probleme war bisher das Fehlen eines guten Tiermodells der Parkinsonkrankheit.

Während der letzten fünf Jahre hat das Neurotoxin MPTP (1-Methyl-4-phenyl-1,2,3,6-tetrahydropyridin) mit seiner in hohem Maße selektiven Toxizität für dopaminerge Neurone bei der Erforschung der idiopathischen Parkinsonkrankheit große Bedeutung erlangt, weil es sowohl beim Menschen als auch bei anderen Primaten ein Parkinsonsyndrom erzeugt.

\section{Parkinsonsyndrom beim Menschen durch MPTP}

Im Jahre 1979 erschien ein Fallbericht über einen Studenten, der nach Drogenmißbrauch ein anhaltendes Parkinsonsyndrom entwickelte (Davis et al., 1979). Dieser Patient war abhängig von Pethidin und hatte bei der Suche nach stärker wirksamen Derivaten versucht, MPPP (1-Methyl-4phenyl-4-propionoxipiperidin) herzustellen. Nach mehrfacher erfolgreicher Synthese veränderte er die Reaktionsbedingungen, um die Synthesezeit zu verkürzen. Nachdem er sich das Produkt dieser neuen Prozedur zugeführt hatte, zeigte er Akinesie, Rigor und Tremor. Die Behandlung des Parkinsonsyndroms bei diesem Patienten mit L-Dopa und später mit dem Dopaminagonisten Bromokriptin führte zu einer merklichen Verbesserung seines Zustandes. Liquoruntersuchungen deuteten auf Veränderungen des Dopamingehalts im Gehirn hin, da die Liquorkonzentration des Dopaminmetaboliten Homovanillinsäure deutlich vermindert war, während serotonerge und noradrenerge
Funktionen nicht beeinträchtigt schienen. Der von Davis et al. (1979) beschriebene Patient verstarb später an einer Drogenüberdosierung. Die neuropathologische Untersuchung zeigte massiven Neuronenverlust und Gliose in der Pars compacta der Substantia nigra; der Locus coeruleus war nicht verändert. Obgleich man vermutete, daß MPPP oder einige Derivate Ursache des Parkinsonsyndroms dieses Patienten seien, wurde die toxische Substanz nicht identifiziert.

Im Jahre 1983 berichteten Langston und Mitarb. über vier weitere junge kalifornische Drogenabhängige mit Parkinsonsyndrom, die sich als synthetisches Heroin verkaufte pethidin-analoge Substanzen intravenös injiziert hatten. Die Symptome dieser Patienten besserten sich nach der Gabe von LDopa oder Dopaminagonisten. Zwei Patienten hatten unterhalb des Normalbereichs liegende Homovanillinsäure-Konzentrationen im Liquor. Alle vier Patienten hatten sich MPPP verabreicht. Allerdings erschien es unwahrscheinlich, daß MPPP das verursachende Toxin war, da der von Davis et al. (1979) beschriebene Patient zunächst einige Monate lang reines MPPP eingenommen hatte, ohne daß er erkrankte. Die chemische Analyse der von den Drogenabhängigen injizierten Substanz ergab, daß sie in unterschiedlichem Maße MPTP (1-Methyl-4-phenyl1,2,3,6-tetrahydropyridin) enthielt und daß diese Substanz für die Entstehung des Parkinsonsyndroms verantwortlich war. Später wurden in Kalifornien etwa 400 Drogenkonsumenten entdeckt, die sich MPTP in der Annahme, es sei Heroin, injiziert hatten (Ruttenber et al., 1986).

Im Hinblick auf Symptomatik und körperlichen Untersuchungsbefund gleichen Personen mit Parkinsonsyndrom, das durch MPTP hervorgerufen wird, in hohem Maße Patienten mit idiopathischer Parkinsonkrankheit (Ballard et al., 1985). Ein Unterschied besteht im Alter bei Beginn der Erkrankung. Während die Drogenabhängigen ihr Parkinsonsyndrom im Alter von 20 bis 40 Jahren entwickelten, liegt der Krankheitsbeginn bei idiopathischer Parkinsonkrankheit häufig zwischen dem 50. und 60. Lebensjahr. Alle klassischen Kennzeichen der idiopathischen Form sind bei Patienten mit MPTP-Parkinsonsyndrom zu beobachten, nämlich langsamer Ablauf aller Bewegungen und Reduktion der spontanen Bewegungen (Akinesie) mit starrer Mimik, Drehen des Kopfes ,en bloc" zusammen mit den Schultern und dem Rumpf, Fehlen der Mitbewegung der Arme beim Gehen und Mikrographie, weiterhin erhöhter Muskeltonus (Rigor) und schließlich Ruhetremor. Wie bei der idiopathischen Parkinsonkrankheit sind auch bei der MPTP-Erkrankung kognitive Defizite zu beobachten (Stern und Langston, 1985).

Hinsichtlich der pharmakologischen Therapie sind die Ähnlichkeiten zwischen MPTP-induziertem Parkinsonsyndrom und idiopathischer Krankheit nicht weniger bemerkenswert. Amantadin und Anticholinergika sind für MPTPKranke nur von sehr geringem Nutzen, was die geringe Wirksamkeit dieser Pharmaka bei mittlerer und schwerer Parkinsonkrankheit widerspiegelt (Ballard et al., 1985). L-Dopa in Kombination mit dem Dekarboxylasehemmer Karbidopa hingegen zeigt deutliche therapeutische Erfolge (Ballard et al., 1985; Langston und Ballard, 1984). Bei den MPTP-Patienten treten auch die für eine längere Behandlungszeit charakteristischen Nebenwirkungen der L-Dopa-Therapie auf, wie Dyskinesien, „,On-OffFluktuationen" und Halluzinationen. 


\section{Umwelttoxine und Parkinsonkrankheit}

Die Ursache der idiopathischen Parkinsonkrankheit ist unbekannt. Untersuchungen an eineiigen und zweieiigen Zwillingen lassen keine genetische Prädisposition für die Erkrankung erkennen (Ward et al., 1983; Marsden, 1987). Daher ist eine Beteiligung von Umweltfaktoren wahrscheinlich. Belege für eine ätiologische Bedeutung von Viren gibt es nicht (Duvoisin, 1982). Daß ein Umwelttoxin die Ursache der Parkinsonkrankheit sein kann, wird seit vielen Jahren in Erwägung gezogen. Die Entdeckung eines vergleichsweise einfachen Moleküls mit ausgeprägter Toxizität für die Substantia nigra verschafft dieser Hypothese zweifellos neuen Auftrieb.

Die Bedeutung des Kontakts mit MPTP in der Umgebung wird durch den Fall eines Chemikers belegt, der mit MPTP arbeitete und im Alter von 38 Jahren an Parkinsonsyndrom litt (Langston und Ballard, 1983). Er verwendete die Substanz als Zwischenstufe bei der Synthese von Analgetika. Die einzigen Möglichkeiten der Zufuhr von MPTP waren Hautkontakt oder Inhalation. Weitere Parkinsonerkrankungen bei Chemikern, die für legitime Zwecke MPTP verwendeten, wurden entdeckt, und Parkinsonsymptome waren bei Industriearbeitern, die MPTP ausgesetzt waren, deutlich häufiger als bei Personen ohne entsprechende Exposition (Barbeau et al., 1985). Ob nun MPTP in der Umwelt die idiopathische Parkinsonkrankheit verursacht, ist bislang ungeklärt. Es liegen keine Belege für das Vorhandensein dieses Stoffes in der natürlichen oder der vom Menschen geschaffenen Umwelt vor. Allerdings wurden auch noch keine systematischen Untersuchungen in dieser Richtung durchgeführt.

Ein Hinweis für das Vorkommen von Neurotoxinen in der Natur stammt von einer Krankheit, die anscheinend nur Pferde befällt (Mettler und Stern, 1962). Nigro-pallidale Enzephalomalazie wird beobachtet, wenn Pferde große Mengen einer bestimmten Mistelart (Centaurea solstitialis) fressen. Erkrankte Tiere entwickeln parkinsonähnliche Symptome, sie sind rigide und haben Freß- und Schluckbeschwerden. Pathologisch zeigen sich deutlich abgegrenzte nekrotische Bezirke in der Substantia nigra und im Globus pallidus. Extrakte der Disteln wirken auch bei Labortieren neurotoxisch. Die neurotoxische Substanz und der Wirkmechanismus sind bisher unbekannt. Auch wenn sich die neuropathologischen Befunde bei dieser Pferdekrankheit von der neurotoxischen Wirkung von MPTP unterscheiden, zeigt sich doch, daß Toxine in der Umwelt selektiv Strukturen der Basalganglien schädigen können.

Eine gewisse Ähnlichkeit mit MPTP in der chemischen Struktur hat das Pestizid Paraquat. Eine epidemiologische Studie aus der kanadischen Provinz Quebec zeigt die höchste Inzidenz der Parkinsonkrankheit in landwirtschaftlichen Regionen, in denen Paraquat verwendet wird (Barbeau et al., 1986). Gegen einen weiterreichenden Einfluß von Pestiziden und Herbiziden spricht die Tatsache, daß die agrochemische Industrie während der letzten Jahrzehnte rapide angewachsen ist, während die Inzidenz der Parkinsonkrankheit seit der Jahrhundertwende gleich geblieben zu sein scheint (Duvoisin, 1982).

Nicht auszuschließen ist schließlich die Hypothese, daß ein unbekanntes Toxin mit der Nahrung zugeführt wird. Pyridinderivate, die MPTP nicht unähnlich sind, entstehen beim Kochen von Zuckerlösungen. Der resultierende Karamel, eine komplexe Mischung aus zahlreichen Bestandteilen, wird in der Nahrungsmittelindustrie häufig als Aromastoff oder Färbemittel verwendet.

\section{Wirkung von MPTP bei Tieren}

Da MPTP beim Menschen Symptome und pathologische Veränderungen der idiopathischen Parkinsonkrankheit erzeugt, vermutete man bald, daß das Toxin auch für die Entwicklung eines Tiermodells der Krankheit brauchbar sei. Vor der Entdeckung der Neurotoxizität von MPTP war kein gutes Tiermodell vorhanden. Einerseits gab es Ansätze, die beispielsweise durch Reserpingabe bei Versuchstieren den Dopamingehalt des Gehirns erschöpfen und die motorische Aktivität der Tiere zeitweise vermindern. Zwar reagieren Nagetiere, die auf diese Weise akinetisch werden, auf viele Pharmaka, die auch bei der Parkonsonkrankheit wirksam sind, aber abgesehen von der Bewegungsverminderung können die neuropathologischen und ausgedehnten pathochemischen Befunde der Krankheit nicht nachgebildet werden (Carlsson et al., 1957; Dolphin et al., 1976). Andererseits versuchte man, stereotaktisch die Substantia nigra zu zerstören. Ratten mit neurochemischer Läsion der Substantia nigra wurden als Modell für die Entwicklung neuer Antiparkinsonmittel verwendet (Reavill et al., 1983). Bei Affen wurden elektrolytisch oder thermisch Hirnstammläsionen verursacht, um Parkinsonsymptome zu erzeugen (Goldstein et al., 1973; Pechadre et al., 1976). Entscheidende Nachteile dieser Modelle sind die geringe Ähnlichkeit der pathologischen und biochemischen Änderungen mit der Parkinsonkrankheit und die große Variation der motorischen Störungen bei verschiedenen Tieren aufgrund der Schwierigkeit, die Gehirnläsionen präzise zu setzen.

Zahlreiche Untersuchungen zeigen, daß MPTP nicht nur beim Menschen dopaminerge Neurone der Substantia nigra zerstört, sondern auch bei einigen Tierarten. Mäuse sind empfindlich gegenüber den Wirkungen von MPTP, Ratten hingegen nur in sehr geringem Maße (Boyce et al., 1984; Chiueh et al., 1984; Heikkila et al., 1984 a, b; Welzl und Lange, 1986). Bei Mäusen findet man nach MPTP-Gabe nigralen Neuronenverlust und striatalen Dopaminverlust, aber keine offensichtlichen parkinsonähnlichen Symptome (Heikkila et al., 1984 a, b; Maver et al., 1986 b). Ratten zeigen selbst bei intranigraler MPTP-Applikation nur geringfügige Dopaminerniedrigung (Bradhury et al., 1986) und kurzfristige Verhaltensänderungen (Lange und Welzl, 1987).

Die Gabe von MPTP bei Affen führt zu drei aufeinanderfolgenden Phasen veränderter motorischer Aktivität (Burns et al., 1983; Jenner et al., 1984; Langston et al., 1984a). Während der akuten Phase, 30 bis 60 Minuten nach MPTPGabe, scheinen die Tiere einzuschlafen, und sie fallen von den Sitzstangen ihrer Käfige. Häufig treten dystone Haltungen des Rumpfes und der Extremitäten oder wildes Umherrennen auf. Diese akuten Effekte sind möglicherweise durch die Freisetzung verschiedener Monoamine des Gehirns verursacht. In der subakuten Phase während der ersten Wochen nach der MPTP-Gabe entwickeln sich andauernde motorische Einschränkungen. Die Affen werden zunehmend akinetisch, sind rigide in den Extremitäten und weisen Haltungsänderungen auf. Tremor tritt nicht bei allen Tieren auf; es handelt sich eher um einen Haltungstremor als um einen Ruhetremor. Diese Verhaltensänderungen sind einige Wochen lang beobachtbar; danach erholen sich die Affen langsam. In den folgenden Wochen der chronischen Phase stabilisieren sich die motorischen Defizite. Die Affen können sich frei bewegen, zeigen aber im Vergleich zu unbehandelten Tieren we- 
niger Spontanbewegungen. Komplizierte Bewegungen sind schlecht koordiniert und werden unbeholfen ausgeführt. Die Brandbreite an Bewegungen ist deutlich eingeschränkt. Alle beschriebenen motorischen Defizite sind durch die Gabe von LDopa zusammen mit einem peripheren Dekarboxylase-Hemmer wie auch durch synthetische Dopaminagonisten behandelbar.

Das MPTP-Parkinsonsyndrom der Affen unterscheidet sich von der idiopathischen Parkinsonkrankheit durch das geringe Auftreten von Tremor, die Art des Tremors und die Erholung von den motorischen Veränderungen bei den Affen. Ähnlichkeiten zwischen neuropathologischen Veränderungen der mit MPTP behandelten Affen und den Befunden post mortem bei Patienten mit idiopathischer Parkinsonkrankheit bestehen in der Zerstörung dopaminerger Neurone in der Substantia nigra (Waters et al., 1986). Schädigungen des Locus coeruleus werden bei den Affen im Gegensatz zur Parkinsonkrankheit nicht beobachtet (Langston et al., 1984a). Auch Lewykörper sind beim Tiermodell nicht vorhanden. Die MPTP-Gabe führt bei Affen zu starken Verlusten an Dopamin in Nucleus caudatus und Putamen (Burns et al., 1983; Jenner et al., 1986a, b; Elsworth et al., 1987). Neuronale Systeme, die Noradrenalin, Serotonin, Azetylcholin, Gamma-Amino-Buttersäure oder Peptide als Überträgerstoffe verwenden, sind nicht einheitlich verändert (Garvey et al., 1986; Jenner et al., 1986 a, b, c). Verglichen mit der idiopathischen Parkinsonkrankheit weist das Affenmodell also begrenzte morphologische und biochemische Änderungen auf.

Zusammenfassend läßt sich sagen, daß das mit MPTP erzeugte Parkinsonsyndrom bei Affen die idiopathische Parkinsonkrankheit in hohem $\mathrm{Maße}$, aber nicht vollständig nachahmt. Die Verhaltensänderungen des Tiermodells gleichen der Krankheit, aber es kommt nach einiger Zeit zu einer gewissen Erholung der motorischen Funktionen. Morphologische und biochemische Befunde der Affen zeigen im Vergleich mit Parkinsonpatienten eine stärker selektive und begrenzte Hirnschädigung. Allerdings liefert die Behandlung von Affen mit MPTP das gegenwärtig beste Tiermodell der Parkinsonkrankheit.

Bei der Bewertung der Unterschiede zwischen Krankheit und Modell ist zu berücksichtigen, daß MPTP den Tieren über einen kurzen Zeitraum zugeführt und der Neuronentod akut herbeigeführt wird, während sich die Entwicklung der Parkinsonkrankheit über viele Jahre erstreckt. In den meisten MPTP-Studien werden junge Affen benutzt; die Parkinsonkrankheit hingegen ist eher eine Krankheit des alternden Gehirns. Neuere Untersuchungen zeigen, daß alte Affen deutlich empfindlicher gegenüber MPTP sind und daß bei diesen Tieren nach MPTP-Gabe auch Neurone des Locus coeruleus zerstört werden und intrazelluläre eosinophile Einschlüsse auftreten (Forno et al., 1986).

Da die motorischen Einschränkungen der mit MPTP behandelten Affen durch L-Dopa zusammen mit einem peripher wirksamen Dekarboxylase-Hemmer oder durch synthetische Dopaminagonisten wie Bromokriptin therapiert werden können (Burns et al., 1983; Jenner et al., 1984; Langston et al., 1984 a), sollte dieses Tiermodell ein geeignetes Mittel für die Überprüfung neuer Antiparkinsonmittel sein. Die Verwendung des Tiermodells zeigt, daß eine Vielzahl von Dopaminagonisten, die selektiv Dopamin-2-Rezeptoren stimulieren, die motorischen Defizite nach MPTP-Applikation ausgleichen kann ( $\mathrm{No}$ moto et al., 1986). Weiterhin kann mit dem Modell die Bedeu- tung von Dopamin-1- und Dopamin-2-Rezeptoren bei der Parkinsonkrankheit untersucht werden. Der selektive Dopamin-1Agonist SFK 38393 beispielsweise kann die motorischen Störungen bei Marmosetten, die MPTP erhalten, nicht verbessern und scheint das Parkinsonsyndrom eher zu verstärken (Nomoto et al., 1985). Dieser tierexperimentelle Befund stimmt mit klinischen Erfahrungen bei der Verwendung von SFK 38393 bei Parkinsonkranken überein (Braun et al., 1987). Außerdem kann das Tiermodell für die Untersuchung der Nebenwirkungen, die bei der Langzeitbehandlung mit L-Dopa auftreten, wertvoll sein, weil mit MPTP behandelte Affen nach wiederholter L-Dopa-Gabe Dyskinesien entwickeln (Bédard et al., 1986). Neue Ansätze, die die Transplantation nigraler Zellen von Feten als Therapie der Parkinsonkrankheit vorsehen, werfen unter anderem Fragen nach dem Ausmaß funktioneller Erholung, der besten intrazerebralen Lokalisation des Transplantats und der Notwendigkeit immunsuppressiver Maßnahmen auf. Möglicherweise kann das MPTP-Tiermodell zur Lösung dieser Probleme beitragen (Redmond et al., 1986; Oertel et al., 1987).

\section{Wirkungsweise von MPTP}

Die Frage nach der Wirkungsweise von MPTP beinhaltet im wesentlichen drei Probleme. Zunächst stellt sich die Frage, auf welche Weise MPTP den Neuronentod in der Substantia nigra verursacht. Weiterhin ist zu klären, warum der Stoff selektiv toxisch für die Substantia nigra ist. Und schließlich bleibt die Frage zu beantworten, weshalb Primaten deutlich empfindlicher gegenüber dem Toxin sind als niedere Tierarten wie zum Beispiel Nager. Die Untersuchung dieser Fragen zum Wirkungsmechanismus von MPTP kann möglicherweise Einblick in die Faktoren geben, die dem Untergang nigraler Neurone bei der Parkinsonkrankheit zugrundeliegen.

Eine wichtige Beobachtung ist zunächst, daß nach MPTP-Gabe eine weitere Substanz, nämlich $\mathrm{MPP}^{+}$(1Methyl-4-phenylpyridin), in hoher Konzentration in Primatengehirnen vorkommt (Langston et al., 1984c; Markey et al., 1984). Während MPTP rasch aus dem Primatengehirn verschwindet, wird $\mathrm{MPP}^{+}$angehäuft und mehrere Tage lang gespeichert (Johannessen et al., 1985). Im Gegensatz dazu werden bei Nagetieren sowohl MPTP als auch MPP ${ }^{+}$rasch aus dem Gehirn entfernt. Die Ursache dafür ist unbekannt. $\mathrm{MPP}^{+}$wird in einigen Regionen des Primatengehirns einschließlich der Substantia nigra akkumuliert, und diese Anhäufung von $\mathrm{MPP}^{+}$führt zu den neurotoxischen Effekten.

Ein weiterer wichtiger Befund betrifft die Wirkung von Monoaminoxidase-(MAO-)Hemmern auf die Anhäufung von $\mathrm{MPP}^{+}$. Wenn Primaten mit dem MAO-Hemmstoff Pargilin vorbehandelt sind, so ist die $\mathrm{MPP}^{+}-$Akkumulation im Gehirn stark herabgesetzt (Cohen et al., 1984; Langston et al., 1984 b; Markey et al., 1984). Die Vorbehandlung mit Pargilin hemmt auch die neurotoxische Wirkung; dabei wird sowohl die Neuronenschädigung in der Substantia nigra pars compacta als auch die Dopaminreduktion in Nucleus caudatus und Putamen verhindert. Interessanterweise verhindert auch Deprenyl, das nur MAO B und nicht MAO A hemmt, die biochemischen Änderungen nach MPTP-Gabe (Cohen et al., 1984). Weitere Beweise für den Zusammenhang zwischen der durch MPTP verursachten Neurotoxizität, der Bildung von $\mathrm{MPP}^{+}$und dem Einfluß von MAO stammen aus Untersuchungen, die die Unterdrückung der MPTP-Toxizität für das nigrostriatale System von Mäusen (Heikkila et al., 1984c) und das Mesenzephalon von Rattenem- 
bryonen (Mytilineou und Cohen, 1984) nach der Gabe von MAO-Blockern nachweisen. Außerdem zeigt sich, daß MPTP ein Substrat für MAO B ist. MPTP wird in Mitochondrien des Gehirns zum Zwischenprodukt MPDP (1-Methyl-4-phenyl-2,3dihydropyridin) oxidiert, welches dann unter enzymatischem Einfluß oder durch Auto-Oxidation zu MPP ${ }^{+}$verwandelt wird (Chiba et al., 1984; Salach et al., 1984). Diese Reaktionsfolge wird durch einen nichtselektiven MAO-Inhibitor wie Pargilin und einen selektiven MAO-B-Inhibitor wie Deprenyl gehemmt, während ein MAO-A-Inhibitor wie Clorgilin nur eine geringe hemmende Wirkung hat. Die Umwandlung von MPTP zu $\mathrm{MPP}^{+}$findet nicht in Neuronen statt, sondern möglicherweise in der Neuroglia (Javitch et al., 1985). MPP $^{+}$geht Bindungen mit Neuromelanin ein, was zur intrazellulären Akkumulation und Neurotoxizität beitragen kann ( $D^{\prime}$ Amato et al., 1986). Limitierende Faktoren für die Entfaltung der MPTP-Toxizität können somit unterschiedliche Verteilungen von MAO A und MAO B bei verschiedenen Tierarten (Willoughby et al., 1987) sowie das Vorhandensein oder Fehlen von Neuromelanin in verschiedenen Hirnregionen sein.

$\mathrm{MPP}^{+}$, nicht aber MPTP, ist ein Substrat der zellulären Aufnahme von Dopamin und wird in vergleichbarem Umfang wie Dopamin aufgenommen (Javitch und Synder, 1985). Substanzen, die die zelluläre Aufnahme von Dopamin blockieren, verhindern auch die Aufnahme von $\mathrm{MPP}^{+}$in striatale Synaptosomen der Ratte. Kortikale Synaptosomen der Ratte, in denen Aufnahmemechanismen für Noradrenalin vorherrschen, zeigen nur geringe $\mathrm{MPP}^{+}$-Aufnahme. Die Verabreichung von Mazindol, das die Wiederaufnahme von Dopamin blokkiert, verhindert bei Mäusen die toxische Wirkung von MPTP (Heikkila et al., 1985; Ricaurte et al., 1985). Die Beteiligung von Aufnahmemechanismen stellt einen weiteren limitierenden Faktor für die toxischen Wirkungen von MPTP und $\mathrm{MPP}^{+}$dar.

Auf welche Weise MPP ${ }^{+}$innerhalb der Neuronen den Zelltod herbeiführt, ist ungeklärt. Mögliche Mechanismen sind die Beeinflussung mitochondrialer Oxidation (Heikkila et al., 1985) oder zellulärer Redoxreaktionen (Johannessen et al., 1985).

Der bisherige Kenntnisstand über die Wirkungsweise von MPTP läßt sich folgendermaßen zusammenfassen. MPTP wird nach der Aufnahme in das Gehirn extraneuronal durch MAO B in $\mathrm{MPP}^{+}$umgewandelt. $\mathrm{MPP}^{+}$wird dann in dopaminerge Neurone aufgenommen, wird dort akkumuliert und verursacht auf unbekannte Weise den Zelltod. Die Toxizität von MPTP kann entweder durch Blockade von MAO B (z. B. Deprenyl) oder durch Hemmung der Wiederaufnahme von Dopamin (z. B. Mazindol) unterdrückt werden.

\section{Therapeutische Konsequenzen}

Unter der Voraussetzung, daß die Wirkungsweise von MPTP pathogenetische Prozesse der idiopathischen Parkinsonkrankheit widerspiegelt, könnten klinische Pharmakostudien zeigen, ob Substanzen, die die Toxizität von MPTP bei Tieren aufheben, auch bei der Parkinsonkrankheit erfolgreich sind. Die Tatsache, daß MPP ${ }^{+}$ein Substrat des Aufnahmemechanismus für Dopamin ist, könnte die Grundlage für den therapeutischen Versuch von Aufnahmeblockern wie Mazindol oder Nomifensin sein (Mayer et al., 1986a; Schultz et al., 1986). Der Befund, daß durch MAO-Blockade die experimentelle Erzeugung eines Parkinsonsyndroms verhindert wird, läßt an die
Möglichkeit denken, mit MAO-inhibierenden Substanzen den fortschreitenden Neuronenuntergang in der Substantia nigra zu verlangsamen.

MAO-B-Inhibitoren wie L-Deprenyl und Pargilin hemmen die neurotoxische Wirkung von MPTP (Heikkila et al., 1984 c). Langzeituntersuchungen bei der Parkinsonkrankheit liegen für L-Deprenyl vor (Birkmayer et al., 1983, 1985). Die retrospektiven Analysen von Birkmayer und Mitarb. zeigen, da 3 L-Deprenyl in Kombination mit L-Dopa und einem Dekarboxylasehemmer die Wahrscheinlichkeit, daß Parkinsonpatienten ein hohes Lebensalter erreichen, erhöht im Vergleich mit einer Behandlung mit L-Dopa und Dekarboxylasehemmer. Das Fortschreiten der Krankheit wird allerdings nur verzögert und nicht beendet. Der Befund, daß L-Deprenyl-Gabe in niedriger Dosierung zusätzlich zur klassischen Therapie die Lebenserwartung der Parkinsonkranken erhöht, bedarf der Bestätigung durch eine randomisierte kontrollierte Studie. Zumindest scheinen Nebenwirkungen der Langzeitgabe von L-Deprenyl ausgeschlossen zu sein. Auch erscheinen Versuche sinnvoll, die L-Deprenyl während der frühen Stadien der Parkinsonkrankheit oder sogar im subklinischen Stadium - falls ein solches identifiziert werden kann - testen.

Im Hinblick auf die Therapie der Parkinsonkrankheit erlaubt das durch MPTP erzeugte Tiermodell voraussichtlich die empirische Überprüfung neuer Behandlungsverfahren, während Untersuchungen zum Wirkungsmechanismus von MPTP von heuristischem Wert sind, da sie zur Prüfung bisher nicht verwendeter Pharmakagruppen führen können.

\section{Literatur}

Ballard, P. A., J.W. Tertrud, J.W. Langston: Permanent human parkinsonism due to 1-methyl-4-phenyl-1,2,3,6-tetrahydropyridine (MPTP): Seven cases. Neurology 35 (1985) 949-956

Barbeau, A., M. Roy, J.W. Langston: Neurological consequence of industrial exposure to 1-methyl-4-phenyl-1,2,3,6-tetrahydropyridine. Lancet i (1985) 747

Barbeau, A., M. Roy, T. Cloutier, L. Plasse, S. Paris: Environmental and genetic factors in the etiology of Parkinson's disease. In: Advances of Neurology, Vol. 45. Eds.: M.D. Yahr, K.J. Bergmann. New York: Raven (1986)

Bédard, P.J., T. DiPaolo, P. Falardeau, R. Boucher: Chronic treatment with L-Dopa, but not bromocriptine induces dyskinesia in MPTPparkinsonian monkeys. Correlation with $\left[{ }^{3} \mathrm{H}\right]$ spiperone binding. Brain Res. 379 (1986) 294-299

Birkmayer, W., J. Knoll, P. Riederer, M.B.H. Youdim: (-)-Deprenyl leads to prolongation of L-Dopa efficacy in Parkinson's diseasc. Mod. Probl. Pharmacopsychiat. 19 (1983) 170-176

Birkmayer, W., J. Knoll, P. Riederer, M. B. H. Youdim, V. Hars, J. Marton. Increased life expectancy resulting from addition of L-deprenyl to Madopar treatment in Parkinson's disease: A longterm study. J. Neural Transm. 64 (1985) 113-127

Boyce, S., E. Kelly, C. Reavill, P. Jenner, C. D. Marsden: Repeated administration of $\mathrm{N}$-methyl-4-phenyl-1,2,5,6-tetrahydropyridine to rats is not toxic to striatal dopamine neurones. Biochem. Pharmacol. 33 (1984) 1747-1752

Bradbury, A.J., B. Costall, A. M. Domeney, P. Jenner, M. E. Kelly, C.D. Marsden, R.J. Naylor: 1-Methyl-4-phenylpyridine is neurotoxic to the nigrostriatal dopamine pathway. Nature 319 (1986) 5657

Braun, A., G. Fabbrini, M.M. Mouradian, C. Serrati, P. Barone. T.N. Chase: Selective D-1 dopamine receptor agonist treatment of Parkinson's disease. J. Neural. Transm. 68 (1987) 41 - 50

Burns, R.S., C.C. Chiueh, S. P. Markey, M. H. Ebert, D. M. Jacobowitz, I.J. Kopin: A primate model of parkinsonism: selective destruction of dopaminergic neurons in the pars compacta of the substantia nigra by N-methyl-4-phenyl-1,2,3,6-tetrahydropyridine. Proc. Natl. Acad. Sci. U.S.A. 80 (1983) $4546-4550$ 
Carlsson, A., M. Lindqvist, T. Magnusson: 3,4-Dihydroxyphenylalanine and 5-hydroxytryptophan as reserpine antagonists. Nature 180 (1957) 1200

Chiba, $K$., A. Trevor, $N$. Castagnoli: Metabolism of the neurotoxic tertiary amine, MPTP, by brain monoamine oxidase. Biochem. Biophys. Res. Commun. 120 (1984) 574-578.

Chiueh, C. C., S. P. Markey, R.S. Burns, J.N. Johannessen, A. Pert, I.J. Kopin: Neurochemical and behavioral effects of systemic and intranigral administration of N-methyl-4-phenyl-1,2,3,6tetrahydropyridine in the rat. Eur. J. Pharmacol. 100 (1984) 189-194

Cohen, G., P. Pasik, B. Cohen, A. Leist, C. Mytilineou, M. D. Yahr: Pargyline and deprenyl prevent the neurotoxicity of 1-methyl-4-phenyl1,2,3,6-tetrahydropyridine (MPTP) in monkeys. Eur. J. Pharmacol. 106 (1984) 209-210

Davis, G.C., A.C. Williams, S.P. Markey, M. H. Ebert, E. D. Caine, C. M. Reichert, I.J. Kopin: Chronic parkinsonism secondary to intravenous injection of meperidine analogues. Psychiat. Res. 1 (1979) $249 \quad 254$

D'Amato, R.J., Z.P. Lipman, S. H. Snyder: Selectivity of the parkinsonian neurotoxin MPTP: Toxic metabolite $\mathrm{MPP}^{+}$binds to neuromelanin. Science 231 (1986) 987-989

Dolphin, A.C., P. Jenner, C.D. Marsden: The relative importance of dopamine and noradrenaline receptor stimulation for the restoration of motor activity in reserpine or $\alpha$-methyl-p-tyrosine pretreated mice. Pharmacol. Biochem. Behav. 4 (1976) $661-670$

Duvoisin, R.C.: The cause of Parkinson's disease. In: Movement disorders Eds.: C.D. Marsden, S. Fahn. London: Butterworths (1982)

Elsworth, J.D., A. Y. Deutch, D.E. Redmond Jr., J.R. Sladek Jr., R. H. Roth: Differential responsiveness to 1-methyl-4-phenyl-1,2,3,6tetrahydropyridine toxicity in sub-regions of the primate substantia nigra and striatum. Life Sci. 40 (1987) 193-202

Forno, L. S.: Pathology of Parkinson's disease. In: Movement disorders. Eds.: C.D. Marsden, S. Fahn. London: Butterworths (1982)

Forno, L.S., J.W. Langston, L.E. DeLanney, I. Irwin, G. A. Ricaurte: Locus coeruleus lesions and eosinophilic inclusions in MPTP-treated monkeys. Ann. Neurology 20 (1986) 449-455

Garvey, J., M. Petersen, C. Waters, S. Rose, S. Hunt, R. Briggs, P. Jenner, C.D. Marsden: Administration of MPTP to the common marmoset does not alter cortical cholinergic function. Movement Disorders 1 (1986) $129-134$

Goldstein, M., A.F. Battista, T. Ohmoto, B. Anagnoste, K. Fuxe: Tremor and involuntary movements in monkeys: Effect of L-DOPA and of a dopamine receptor stimulating agent. Science 179 (1973) 816-817

Heikkila, R.E., F.S. Cabbat, L. Manzino, R.C. Duvoisin: Effects of 1methyl-4-phenyl-1,2,5,6-tetrahydropyridine on neostriatal dopamine in mice. Neuropharmacology 23 (1984a) 711-713

Heikkila, R.E., A. Hess, R.C. Duvoisin: Dopaminergic neurotoxicity of 1-methyl-4-phenyl-1,2,5,6-tetrahydropyridine in mice. Science 224 (1984b) $1451-1453$

Heikkila, R.E., L. Manzino, F.S. Cabbat, R.C. Duvoisin: Protection against the dopaminergic neurotoxicity of 1-methyl-4-phenyl1,2,5,6-tetrahydropyridine by monoamine oxidase inhibitors. Nature $311(1984 \mathrm{c}) 467-469$

Heikkila, R.E., A. Hess, R.C. Duvoisin: Dopaminergic neurotoxicity of 1-methyl-4-phenyl-1,2,5,6-tetrahydropyridine (MPTP) in the mouse: Relationships between monoamine oxidase, MPTP metabolism and neurotoxicity. Life Sci. 36 (1985) 231-236

Hornykiewicz, O.: Brain neurotransmitter changes in Parkinson's disease. In: Movement disorders. Eds.: C. D. Marsden, S. Fahn. London: Butterworths (1982)

Javitch, J.A., S.H. Snyder: Uptake of MPP ${ }^{+}$by dopamine neurons explains selectivity of parkinsonism-inducing neurotoxin MPTP. Eur. J. Pharmacol. 106 (1985) 455-456

Javitch, J. A., R.J. D'Amato, S. M. Strittmatter, S. H. Snyder: Parkinsonism-inducing neurotoxin, N-methyl-4-phenyl-1,2,3,6-tetrahydropyridine: Uptake of the metabolite N-methyl-4-phenylpyridine by dopamine neurons explains selective toxicity. Proc. Natl. Acad. Sci. U.S.A. 82 (1985) 2173-2177

Javoy-Agid, F., H. Taquet, F. Cesselin, J. Epelhaum, D. Grouselle, A. Mauborgne, J. M. Studler, Y. Agid: Neuropeptides in Parkinson's disease. In: Catecholamines, Part C: Neuropharmacology and Central Nervous System - Therapeutic Aspects. Eds.: E. Usdin, A. Carlsson, A. Dahlstum, J. Engel. New York: Alan R. Liss (1984)

Jenner, P., N.M.J. Rupniak, S. Rose, E. Kelly, G. Kilpatrick, A. Lees,
C.D. Marsden: 1-Methyl-4-phenyl-1,2,3,6-tetrahydropyridineinduced parkinsonism in the common marmoset. Neurosci. Lett. 50 (1984) $85-90$

Jenner, P., C.D. Marsden, B. Costall, R.J. Naylor: MPTP and MPP ${ }^{+}$ induced neurotoxicity in rodents and the common marmoset as experimental models for investigating Parkinson's disease. In: MPTP: A neurotoxin producing a parkinsonian syndrome. Eds.: S.P. Markey, N. Castagnoli Jr., A.J. Trevor, I.J. Kopin. New York: Academic Press (1986a)

Jenner, P., S. Rose, M. Nomoto, C.D. Marsden: MPTP-induced parkinsonism in the common marmoset: Behavioural and biochemical effects. In: Advances of Neurology, Vol. 45. Eds.: M.D. Yahr, K.J. Bergmann. New York: Raven Press (1986 b)

Jenner, P., H. Taquet, A. Mauborgne, J. T. Benoliel, F. Cesselin, S. Rose, F. Javoy-Agid, Y. Agid, C. D. Marsden: Lack of change in basal ganglia neuropeptide content following subacute 1-methyl-4-phenyl1,2,3,6-tetrahydropyridine treatment of the common marmoset. J. Neurochem. 47 (1986c) 1548-1551

Johannessen, J. N., C.C. Chiueh, R. S. Burns, S.P. Markey: Differences in the metabolism of MPTP in the rodent and primate parallel differences in sensitivity to its neurotoxic effects. Life Sci. 36 (1985) 219-224

Lange, $K . W ., H$. Welzl: Acute behavioral effects of unilateral intranigral injection of MPTP in the rat. Neuroscience 22 (1987) S359

Langston, J.W., P. A. Ballard: Parkinson's disease in a chemist working with 1-methyl-4-phenyl-1,2,5,6-tetrahydropyridine. N. Engl. J. Med. 309 (19839 310

Langston, J.W., P. Ballard, J.W. Tetrud, I. Irwin: Chronic parkinsonism in humans due to a product of meperidine-analog synthesis. Science 219 (1983) $979-980$

Langston, J. W., P. Ballard: Parkinsonism induced by 1-methyl-4-phenyl1,2,3,6-tetrahydropyridine: Implications for treatment and the pathogenesis of Parkinson's disease. Can. J. Neurol. Sci. 11 (1984) $160-165$

Langston, J.W., L.S. Forno, C.S. Rebert, I. Irwin: Selective nigral toxicity after systemic administration of 1-methyl-4-phenyl-1,2,5,6tetrahydropyridine (MPTP) in the squirrel monkey. Brain Res. 292 (1984 a) 390-394

Langston, J. W., I. Irwin, E. B. Langston, L.S. Forno: Pargyline prevents MPTP-induced parkinsonism in primates. Science 225 (1984b) $1480-1482$

Langston, J.W., I. Irwin, E.B. Langston, L.S. Forno: 1-Methyl-4phenylpyridinium ion $\left(\mathrm{MPP}^{+}\right)$: Identification of a metabolite of MPTP, a toxin selective to the substantia nigra. Neurosci. Lett. 48 (1984c) 87-92

Markey, S. P., J. N. Johannessen, C. C. Chiueh, R. S. Burns, M. A. Herkenham: Intraneuronal generation of a pyridinium metabolite may cause drug-induced parkinsonism. Nature 311 (1984) 464-467

Marsden, C.D.: Parkinson's disease in twins. J. Neurol. Neurosurg. Psychiat. 50 (1987) 105-106

Mayer, R. A., M.V. Kindt, R.E. Heikkila: Prevention of the nigrostriatal toxicity of 1-methyl-4-phenyl-1,2,3,6-tetrahydropyridine by inhibitors of 3,4-dihydroxyphenylethylamine transport. J. Neurochem. 47 (1986a) 1073-1079

Mayer, R.A., A.S. Walters, R.E. Heikkila: 1-Methyl-4-phenyl-1,2,3,6tetrahydropyridine (MPTP) administration to C57-black mice leads to parallel decrements in neostriatal dopamine content and tyrosine hydroxylase activity. Eur. J. Pharmacol. 120 (1986b) 375-377

Mettler, F.A., G.M. Stern: Observations on the toxic effects of yellow star thistle. J. Neuropathol. Exp. Neurol. 22 (1962) 164-169

Mytilineou, C., G. Cohen: 1-Methyl-4-phenyl-1,2,3,6-tetrahydropyridine destroys dopamine neurons in explants of rat embryo mesencephalon. Science 225 (1984) 529-531

Nomoto, M., P. Jenner, C.D. Marsden: The dopamine D-2 agonist LY 141865 , but not the D-1 agonist SKF 38393, reverses parkinsonism induced by 1-methyl-4-phenyl-1,2,3,6-tetrahydropyridine (MPTP) in the common marmoset. Neurosci. Lett. 57 (1985) 37-41

Nomoto, M., P. Jenner, C.D. Marsden: Alterations in motor behaviour produced by the isomers of 3-PPP in the MPTP-treated marmoset. Eur. J. Pharmacol. 121 (1986) 123-128

Oertel, W. H., A. Fine, P. N. Chong, S. Hunt, M. Nomoto, S. Dunnett, L. Annett, P. Jenner, C.D. Marsden: Pilot study of transplantation of foetal nigral cells into striatum of marmosets with MPTP-induced parkinsonian syndrome. Brit. J. Pharmacol. 92 (1987) 611 P

Péchadre, J.C., L. LaRochelle, L.J. Poirier: Parkinsonian akinesia, rigidity and tremor in the monkey. J. Neurol. Sci. 28 (1976) 147-157 
Reavill, C., P. Jenner, C.D. Marsden: Differentiation of dopamine agonists using drug-induced rotation in rats with unilateral or bilateral 6-hydroxydopamine destruction of ascending dopamine pathways. Biochem. Pharmacol. 32 (1983) 865-870

Redmond, D.E., R. H. Roth, J.D. Elsworth, J.R. Sladek Jr., T.J. Collier $A$. Y. Deutch, S. Haber: Fetal neuronal grafts in monkeys given methyl-phenyl-tetrahydropyridine. Lancet i (1986) 1125-1127

Ricaurte, G.A., J.W. Langston, L.E. DeLanney, I. Irwin, J.D. Brooks: Dopamine uptake blockers protect against the dopamine depleting effect of MPTP in the mouse striatum. Neurosci. Lett. 59 (1985) $259-264$

Rinne, U.K.: Brain neurotransmitter receptors in Parkinson's disease. In: Movement disorders. Eds.: C.D. Marsden, S. Fahn. London: Butterworths (1982)

Ruttenber, A.J., P.L. Garbe, H.D. Kalter, K.G. Castro, J.W. Tetrud, P. Porter, I. Irwin, J. W. Langston: Meperidine analog exposure in California narcotics abusers: Initial epidemiologic findings. In: MPTP: A neurotoxin producing a Parkinsonian syndrome. Eds.: S. P. Markey, N. Castagnoli Jr., A.J. Trevor, I.J. Kopin. New York: Academic Press (1986)

Salach, J.I., T.P. Singer, N. Castagnoli Jr., A. Trevor: Oxidation of the neurotoxic amine 1-methyl-4-phenyl-1,2,3,6-tetrahydropyridine (MPTP) by monoamine oxidases $\mathrm{A}$ and $\mathrm{B}$ and suicide inactivation of the enzymes by MPTP. Biochem. Biophys. Res. Comm. 125 (1984) $831-835$

Schultz, W., E. Scarnati, E. Sundstrom, T. Tsutsumi, G. Jonsson: The catecholamine uptake blocker nomifensine protects against MPTP- induced parkinsonism in monkeys. Exp. Brain Res. 63 (1986) $216-220$

Stern, Y., J.W. Langston: Intellectual changes in patients with MPTPinduced parkinsonism. Neurology 35 (1985) 1506-1509

Ward, C.D., R. C. Duvoisin, S. E. Ince, J.D. Nutt, R. Eldridge, D. B. Cal$n e$ : Parkinson's disease in 65 pairs of twins and in a set of quadruplets. Neurology 33 (1983) 815-824

Waters, C. M., S. P. Hunt, A. B. Bond, P. Jenner, C.D. Marsden: Neuropathological, immunohistochemical and receptor changes seen in marmosets treated with MPTP. In: MPTP: A neurotoxin producing a parkinsonian syndrome. Eds.: S.P. Markey, N. Castagnoli Jr., A.J. Trevor, I.J. Kopin. New York: Academic Press (1986)

Welzl, $H ., K . W$. Lange: Unilateral intranigral injection of MPTP in the rat induces contraversive turning. Eur. J. Pharmacol. 132 (1986) 295-297

Willoughby, J., V. Glover, M. Sandler, G. Reynolds: Monoamine oxidase B distribution in the human, marmoset and rat brain. Brit. J. Pharmacol. 92 (1987) 681 P

Dr. K.W. Lange

Department of Neurology

Institute of Psychiatry

Denmark Hill

GB-London SE5 8AF 